\title{
Low-volume polyethylene glycol and bisacodyl for bowel preparation prior to colonoscopy: a meta-analysis
}

\author{
Robert E. Clark, Jonathan D. Godfrey, Abhishek Choudhary, Imran Ashraf, \\ Michelle L. Matteson, Matthew L. Bechtold
}

University of Missouri, Columbia, USA

\begin{abstract}
Background Quality of bowel preparation prior to colonoscopy is essential. Studies have shown a reduced volume of polyethylene glycol (PEG) with bisacodyl may improve visualization and tolerability, but results have varied. Therefore, a meta-analysis was performed to analyze the efficacy of a low-volume PEG bowel preparation with bisacodyl for bowel preparation prior to colonoscopy.

Methods Multiple databases were searched (June 2012). Only randomized controlled trials in peer-reviewed journals on adult subjects comparing low-volume PEG (2 L) with bisacodyl versus 4 L PEG were included. Meta-analysis for the efficacy of low-volume PEG with bisacodyl and $4 \mathrm{~L}$ PEG were analyzed by calculating pooled estimates of number of satisfactory, excellent, and poor bowel preparations as well as adverse patient events (abdominal pain, nausea, vomiting, bloating).

Results Six studies $(\mathrm{N}=1,540)$ met the inclusion criteria. No statistically significant differences were noted between low-volume PEG ( $2 \mathrm{~L}$ ) with bisacodyl and 4 liters PEG for number of satisfactory (OR 0.86; 95\% CI: 0.45-1.63, P=0.64), excellent (OR 1.08; 95\% CI: 0.78-1.50, $\mathrm{P}=0.63$ ), or poor bowel preparations (OR 0.68; 95\% CI: 0.35-1.34, $\mathrm{P}=0.27$ ). A statistically significant decrease in nausea (OR 0.57; 95\% CI: 0.36-0.89, $\mathrm{P}=0.01$ ), vomiting (OR 0.57; 95\% CI: $0.40-0.81, \mathrm{P}<0.01$ ), and bloating (OR 0.65; 95\% CI: 0.49-0.87, $\mathrm{P}<0.01$ ) was noted for the low-volume PEG with bisacodyl as compared to 4 L PEG. No statistically significant differences were noted between the two groups for abdominal pain $(\mathrm{P}=0.62)$.
\end{abstract}

Conclusion Low-volume PEG (2 L) with bisacodyl demonstrates less nausea, vomiting, and bloating without adversely affecting the bowel preparation.

Keywords Polyethylene glycol, bisacodyl, colonoscopy, meta-analysis

Ann Gastroenterol 2013; 26 (4): 319-324

\section{Introduction}

Colonoscopy continues to remain the preferred procedure of investigation of diseases of the colon and terminal ileum. An adequate colonoscopy requires adequate visualization of the colonic and terminal ileum mucosa. Inadequate visualization increases the possibility of missed lesions, prolongs procedure

Division of Gastroenterology, University of Missouri, Columbia, USA

Conflict of Interest: None

Correspondence to: Matthew L. Bechtold MD, FACG, Division of Gastroenterology \& Hepatology, CE405, DC 043.00, University of Missouri Health Sciences Center, Five Hospital Drive, Columbia MO 65212, USA, Tel.:+573 882 1013, Fax: +573 884 4595, e-mail: bechtoldm@health.missouri.edu

Received 20 April 2013; accepted 17 June 2013 time, and increases patient discomfort [1]. In addition, there is an increase in the cost of colonoscopy if the procedure needs to be repeated due to an inadequate preparation [2]. The ideal preparation for colonoscopy would empty the colon of all fecal material without any effect on the histological or endoscopic appearance of the mucosa. Furthermore, it would be palatable to the patient, require a short period of ingestion, cause no patient discomfort, and would have minimal fluid shifts [3].

Since the introduction of polyethylene glycol (PEG) in 1980, PEG solutions have quickly become the preferred method of bowel cleansing [4]. Due to the large volume which is required for adequate cleansing, patient tolerance can be low. Given that over 14 million colonoscopies are performed in the United States annually [5], the costs associated with inadequate bowel preparation can be significant [6]. One of the more important predictors of a poor bowel preparation 
is a patient's inability to complete the preparation due to the large volume which needs to be consumed [6].

In an effort to improve patient tolerance of PEG solutions, several studies have been performed examining low-volume PEG solutions using an adjunct, such as ascorbic acid, magnesium citrate, or bisacodyl [7-11]. Patient compliance, tolerance, and quality of bowel preparation have differed among randomized trails evaluating low-volume PEG and bisacodyl. Therefore, we conducted a meta-analysis to evaluate low-volume (2 L) PEG with bisacodyl versus full-dose (4 L) PEG for bowel preparation prior to colonoscopy.

\section{Materials and methods}

\section{Study selection criteria}

All randomized controlled trials (RCTs) on adult patients comparing large-volume PEG solutions with low-volume PEG solutions and bisacodyl were included in our analysis.

\section{Data collection and extraction}

PubMed, Cochrane Central Register of Controlled Trials \& Database of Systematic Reviews, and CINAHL were searched through June 2012. The search terms were bowel preparations, polyethylene glycol, and bisacodyl. All references from selected

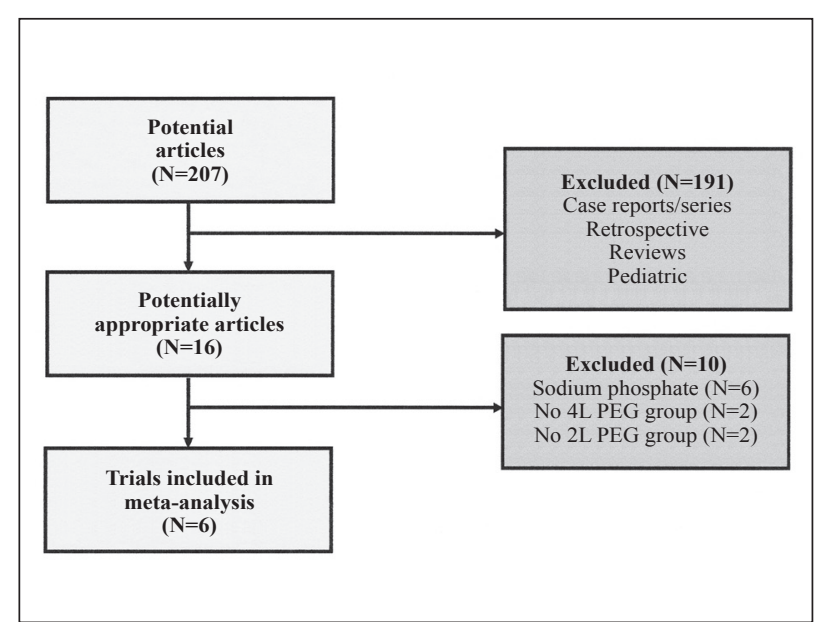

Figure 1 Article search results for this meta-analysis

RCTs were reviewed to ensure no additional trials were omitted from the primary searches. Only RCTs in peer-reviewed journals on adult subjects comparing low-volume PEG (2 L) with bisacodyl versus 4 L PEG were included. Data extraction was performed by two independent reviewers using standard forms. Each study was evaluated by a Jadad score (0-5 with 5 indicating excellent quality and 0 indicating poor quality) [12] and criteria based on Juni et al [13] to assess the quality of the study.

Table 1 Details of studies included in meta-analysis

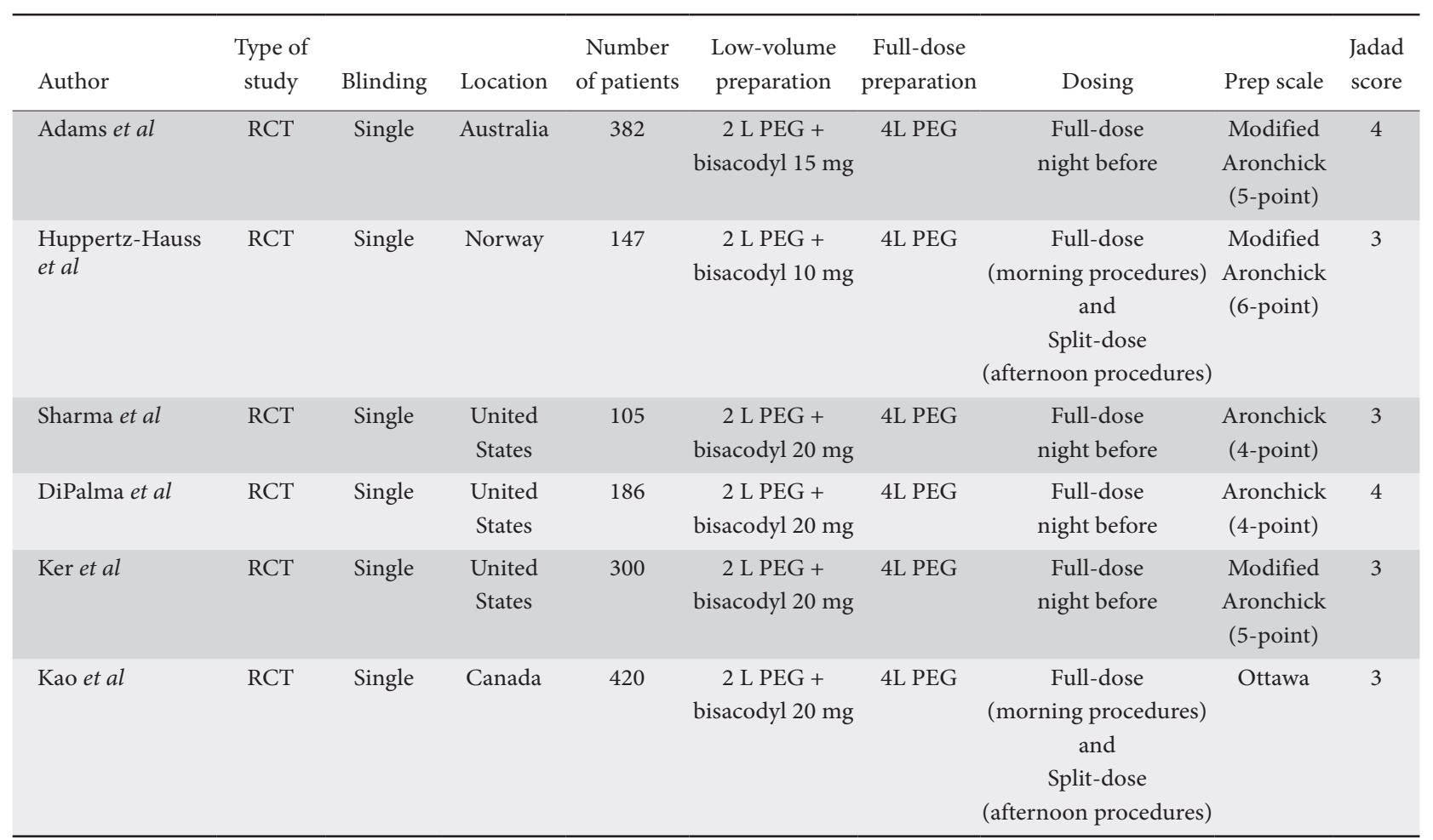

RCT, randomized controlled trial; PEG, polyethylene glycol 


\section{Statistical analysis}

A meta-analysis was performed comparing low-volume PEG with bisacodyl and 4 L PEG for bowel preparation prior to colonoscopy by calculating pooled estimates of quality of bowel preparations (satisfactory, excellent, and poor) as well as adverse patient events (abdominal pain, nausea, vomiting, bloating). Given slightly different bowel preparation scales being used (Aronchick, modified Aronchick, and Ottawa bowel preparation scales), the quality of preparation (satisfactory, excellent, poor) was defined based upon each authors definitions in the individual studies. Separate analyses were performed for each main outcome by using odds ratio (OR) with fixed and random effects models. Heterogeneity among studies was assessed by calculating $\mathrm{I}^{2}$ measure of inconsistency which was considered significant if $\mathrm{P}<0.10$ or $\mathrm{I}^{2}>50 \%$. If heterogeneity was statistically significant, a study elimination analysis was utilized to examine for heterogeneity when certain studies were excluded from the analysis. RevMan 5.1 was utilized for statistical analysis.

\section{Results}

\section{Article search}

The initial search identified 207 articles (Fig. 1). Six studies satisfied the inclusion criteria $(\mathrm{N}=1,540)$ with a mean age ranging from 50 to 63 years. Table 1 shows a summary of the details in each study. All studies used 2 L PEG with bisacodyl with varying dosages and 4 L PEG. Huppertz-Hauss et al used $10 \mathrm{mg}$ of bisacodyl while Adams et al used $15 \mathrm{mg}$ of bisacodyl [14,15]. All other studies used $20 \mathrm{mg}$ of bisacodyl [11,16-18]. A majority of the studies utilized full-dose bowel preparation with all of the preparation taken the night prior to the procedure $[11,14,16,17]$. Two studies did perform split-dose regimen in patients with afternoon appointments only, in which some of the preparation was taken the night before the colonoscopy and the remaining preparation taken the day of the procedure $[15,18]$.

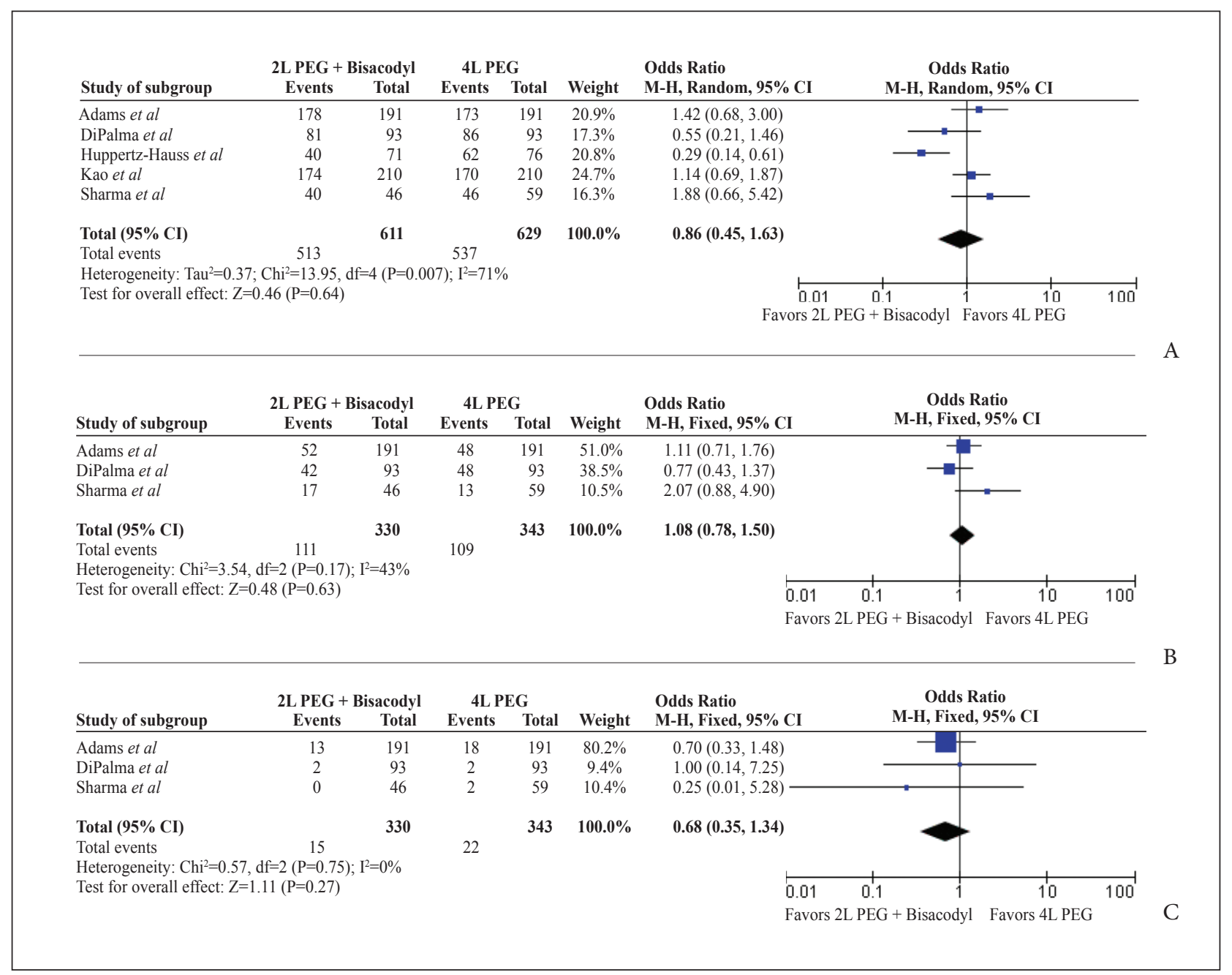

Figure 2 (A) Forest plots for quality of bowel preparation between low-volume polyethylene glycol (PEG) with bisacodyl compared to fulldose PEG for satisfactory, (B) excellent, (C) poor preparation 


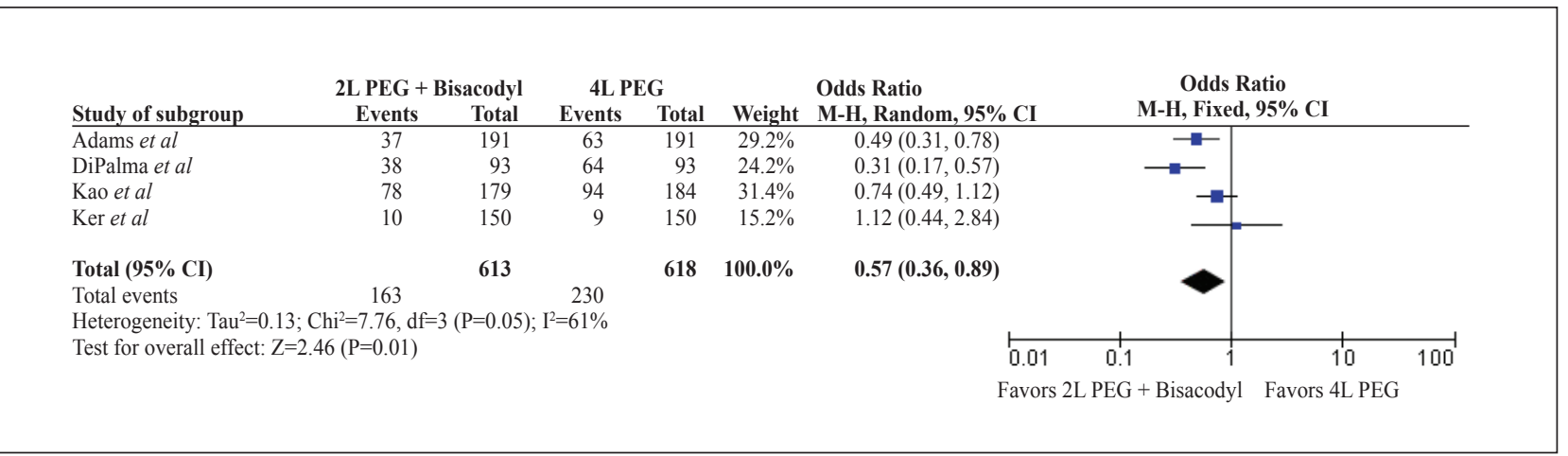

Figure 3 Forest plot for nausea between low-volume polyethylene glycol (PEG) with bisacodyl compared to full-dose PEG

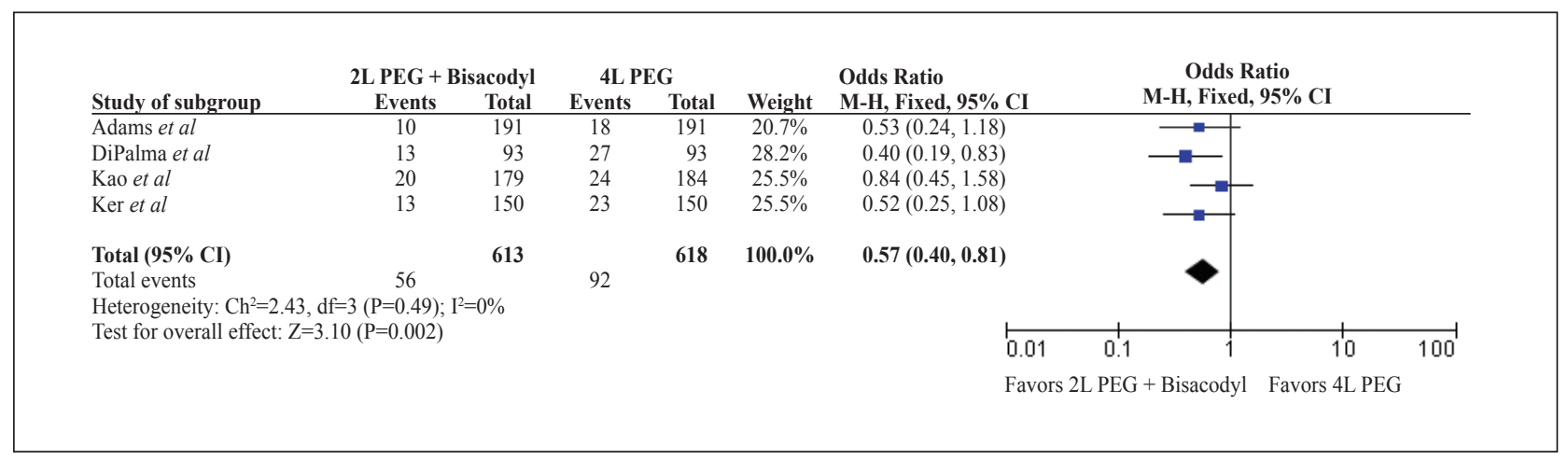

Figure 4 Forest plot for vomiting between low-volume polyethylene glycol (PEG) with bisacodyl compared to full-dose PEG

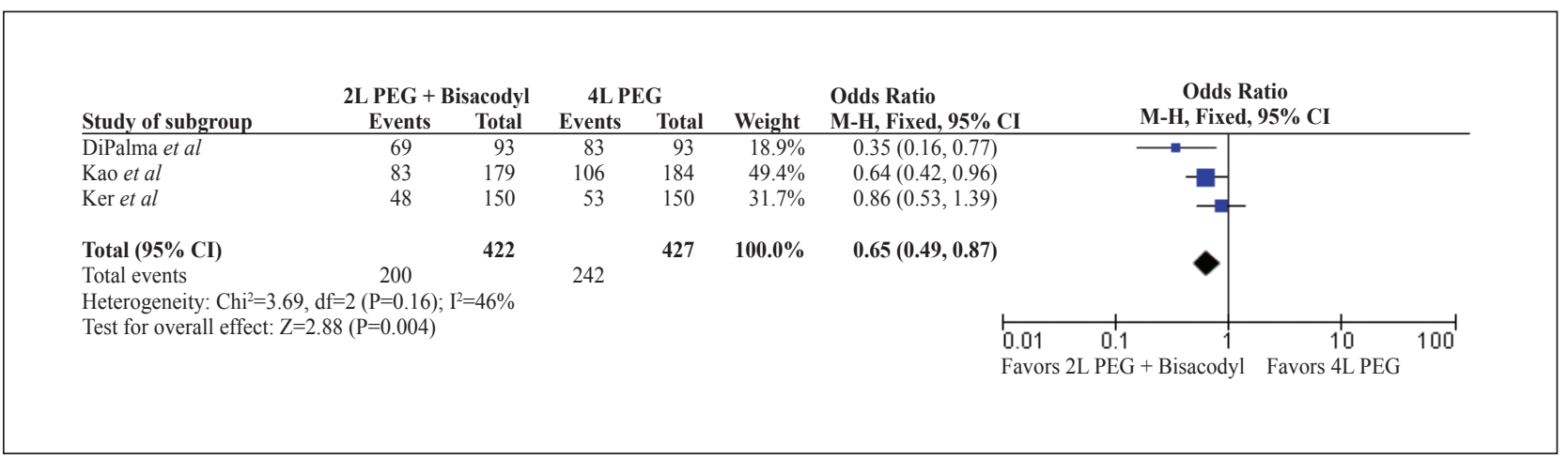

Figure 5 Forest plot for bloating between low-volume polyethylene glycol (PEG) with bisacodyl compared to full-dose PEG

\section{Quality of bowel preparations}

The quality of the bowel prep was examined in five studies [11,14-16,18]. Five studies examined the percentage of patients having satisfactory preps $(\mathrm{N}=1,240)[11,14-16,18]$ while three studies examined excellent and poor preps $(\mathrm{N}=673)[11,14,16]$. There was no statistical difference for the number of satisfactory (OR 0.86; 95\% CI: 0.45-1.63, P=0.64), excellent (OR 1.08; 95\% CI: 0.78-1.50, $\mathrm{P}=0.63$ ), or poor bowel preparations (OR 0.68 ; 95\% CI: 0.35-1.34, P=0.27), (Fig. 2 A-C).

\section{Gastrointestinal side effects}

The frequency of nausea was examined by four studies $(\mathrm{N}=1,231)[14,16-18]$. A statistically significant decrease in the frequency of nausea (OR 0.57; 95\% CI: 0.36-0.89, P=0.01) was noted between the 2 L PEG with bisacodyl as compared to the $4 \mathrm{~L}$ PEG. Fig. 3 shows the Forest plot for nausea.

Four studies examined the side effect of vomiting $(\mathrm{N}=1,231)$ [14,16-18]. A statistically significant decrease in vomiting (OR 0.57; 95\% CI: $0.40-0.81, \mathrm{P}<0.01)$ was observed between the 2 


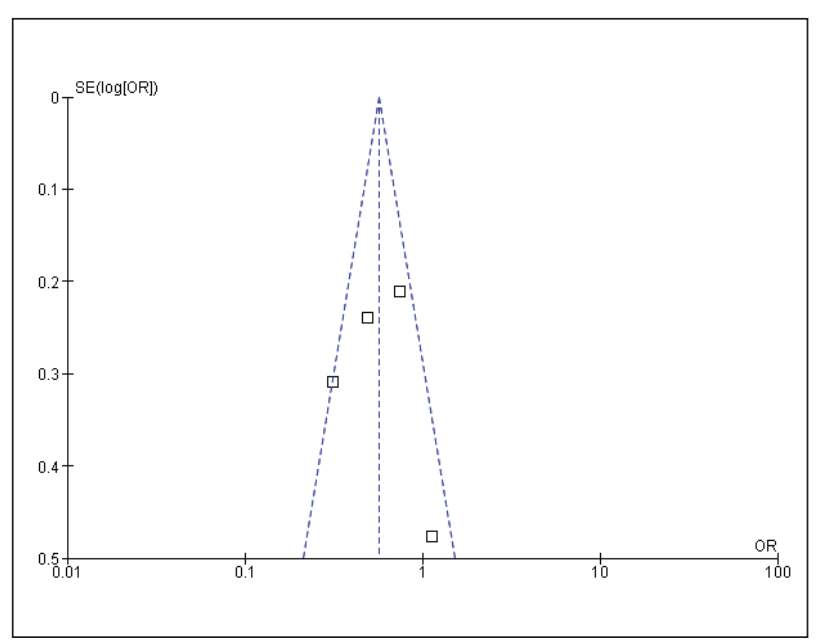

Figure 6 Funnel plot demonstrating no publication bias

L PEG with bisacodyl group compared to the 4 L PEG. Fig. 4 shows the Forest plot for vomiting. No significant heterogeneity was observed $\left(\mathrm{I}^{2}=0 \%, \mathrm{P}=0.83\right)$.

Three studies also examined the frequency of bloating $(\mathrm{N}=849)$ [16-18]. A statistically significant decrease in bloating (OR 0.65; 95\% CI: 0.49-0.87, $\mathrm{P}<0.01$ ) was observed between the 2 L PEG with bisacodyl group compared to the $4 \mathrm{~L}$ PEG (Fig. 5).

Four studies examined the frequency of abdominal pain during the preparation $(\mathrm{N}=1,231)$ [14,16-18]. No statistically significant difference for the frequency of abdominal pain (OR 1.25; 95\% CI: 0.76-2.06, $\mathrm{P}=0.37$ ) was noted between the two groups.

\section{Publication bias}

No significant publication bias was observed for any of the outcomes by the funnel plot (Fig. 6).

\section{Discussion}

The success of a colonoscopy is largely determined by the quality of the bowel preparation. With over 14 million colonoscopies being performed annually in the United States for colon cancer screening purposes alone, the cost of a poor preparation can be substantial $[5,6]$. Patient's tolerance to bowel preparation has mostly been attributed to the large volume required to adequately cleanse the bowel. As many as $38 \%$ of patients do not complete the bowel preparation due to palatability and/or intolerance to the large volume required $[19,20]$. As a result, numerous studies have been performed using a lower volume of PEG solution in an effort to increase tolerability. Several adjuncts have been studied using a smaller volume of PEG solution (2 L), including bisacodyl, senna, magnesium citrate, and ascorbic acid with varying results [7-11,19].

Our meta-analysis was conducted to examine the effects of $2 \mathrm{~L}$ PEG solution with bisacodyl as compared to 4 L PEG. Only RCTs in adult patients were evaluated and used in this study. Based on our results, low-volume PEG (2 L) with bisacodyl (10-20 mg) offers major benefits in clinical practice as it demonstrates less nausea, vomiting and bloating, without adversely affecting the bowel preparation. Therefore, low-volume PEG with bisacodyl may be considered an alternative to the traditional full-dose (4 L) PEG for bowel preparation prior to colonoscopy.

The strengths of this meta-analysis include the use of only RCTs in varying populations and significant endpoints that are applicable to clinical practice. In addition, this represents the first meta-analysis to date on the subject of low-volume PEG with bisacodyl. Limitations to this study are as follows. First, this meta-analysis only addressed low-volume PEG with bisacodyl as compared to full-dose PEG. There are other adjuncts and methods of bowel preparation which are in use and studied, such as senna, vitamin C, magnesium citrate, and split dose preparations, were beyond the scope of this analysis. Second, a limited number of studies were available to be included in this analysis; however, these are the only studies to-date on this topic. Third, the dosage of bisacodyl used varied from 10 to $20 \mathrm{mg}$ depending on the study. Fourth, heterogeneity was noted for two outcomes (satisfactory preparation and nausea). In response, a random effects model was utilized to minimize the heterogeneity effect. Also, a study elimination analysis was performed in which the results were the same without heterogeneity (satisfactory preparation -

OR 1.15; 95\% CI: 0.81-1.64, $\mathrm{P}=0.44 ; \mathrm{I}^{2}=10 \%, \mathrm{P}=0.34$ and nausea - OR 0.65; 95\% CI: $0.49-0.88, \mathrm{P}<0.01 ; \mathrm{I}^{2}=36 \%$, $\mathrm{P}=0.21$ ). Finally, in 2011, the combination of $2 \mathrm{~L}$ PEG and $>5 \mathrm{mg}$ of bisacodyl has been withdrawn from the market in the United States per FDA recommendations due to safety concerns, specifically ischemic colitis. Given this recent event, further randomized controlled trials would have to be performed to evaluate the benefit of 2 L PEG with $5 \mathrm{mg}$ of bisacodyl.

In conclusion, our meta-analysis shows that low-volume (2 L) PEG with bisacodyl demonstrates less nausea and vomiting without adversely affecting the bowel preparation. Therefore, low-volume PEG with bisacodyl appears to be a reasonable alternative to the traditional 4 L PEG for bowel preparation in appropriate patients. However, given the increased risk of ischemic colitis with larger doses of bisacodyl and PEG, additional randomized controlled trials with lowdose bisacodyl and PEG would be extremely beneficial. 


\section{Summary Box}

\section{What is already known:}

- Adequate bowel preparation prior to colonoscopy is extremely important

- Many studies have evaluated alternative bowel preparation prior to colonoscopy to improve tolerability without effecting efficacy of the traditional $4 \mathrm{~L}$ polyethylene glycol (PEG)

- Adding bisacodyl to 2 L PEG may improve tolerability without sacrificing efficacy; however, studies have varied in their results

\section{What the new findings are:}

- 2 L PEG with bisacodyl does improve patient tolerability as compared to the traditional 4 L PEG

- 2 L PEG with bisacodyl demonstrates no statistically significant differences in satisfactory, excellent, or poor bowel preparations prior to colonoscopy as compared to the traditional $4 \mathrm{~L}$ PEG

- 2 L PEG with bisacodyl appears to be a reasonable alternative to the traditional $4 \mathrm{~L}$ PEG in respect to tolerability and efficacy

\section{References}

1. Kim WH, Cho YJ, Park JY, Min PK, Kang JK, Park IS. Factors affecting insertion time and patient discomfort during colonoscopy. Gastrointest Endosc 2000;52:600-605.

2. Rex DK, Imperiale TF, Latinovich DR, Bratcher LL. Impact of bowel preparation on efficiency and cost of colonoscopy. Am J Gastroenterol 2002;97:1696-1700.

3. Tooson JD, Gates LK Jr. Bowel preparation before colonoscopy. Choosing the best lavage regimen. Postgrad Med 1996;100:203204,207-212,214.

4. Davis GR, Santa Ana CA, Morawski SG, Fordtran JS. Development of a lavage solution associated with minimal water and electrolyte absorption or secretion. Gastroenterology 1980;78(5 Pt 1):991-995.

5. Seeff LC, Richards TB, Shapiro JA, et al. How many endoscopies are performed for colorectal cancer screening? Results from CDC's survey of endoscopic capacity. Gastroenterology 2004;127:1670-1677.

6. Ness RM, Manam R, Hoen H, Chalasani N. Predictors of inadequate bowel preparation for colonoscopy. Am J Gastroenterol 2001;96:17971802.

7. Pontone S, Angelini R, Standoli M, et al. Low-volume plus ascorbic acid vs high-volume plus simethicone bowel preparation before colonoscopy. World J Gastroenterol 2011;17:4689-4695.

8. Jansen SV, Goedhard JG, Winkens B, van Deursen CT. Preparation before colonoscopy: a randomized controlled trial comparing different regimes. Eur J Gastroenterol Hepatol 2011;23:897-902.

9. Corporaal S, Kleibeuker JH, Koornstra JJ. Low-volume PEG plus ascorbic acid versus high-volume PEG as bowel preparation for colonoscopy. Scand J Gastroenterol 2010;45:1380-1386.

10. Marmo R, Rotondano G, Riccio G, et al. Effective bowel cleansing before colonoscopy: A randomized study of split-dosage versus non-split dosage regimens of high-volume versus low-volume polyethylene glycol solutions. Gastrointest Endosc 2010;72:313-20.

11. Sharma VK, Chockalingham SK, Ugheoke EA, et al. Prospective, randomized, controlled comparison of the use of polyethylene glycol electrolyte lavage solution in four-liter versus two-liter volumes and pretreatment with either magnesium citrate or bisacodyl for colonoscopy preparation. Gastrointest Endosc 1998;47:167-171.

12. Jadad AR, Moore RA, Carroll D, et al. Assessing the quality of reports of randomized clinical trials: is blinding necessary? Control Clin Trials 1996;17:1-12.

13. Juni P, Altman DG, Egger M. Systematic reviews in health care: Assessing the quality of controlled clinical trials. $B M J$ 2001;323:42-46.

14. Adams WJ, Meagher AP, Lubowski DZ, King DW. Bisacodyl reduces the volume of polyethylene glycol solution required for bowel preparation. Dis Colon Rectum 1994;37:229-233.

15. Huppertz-Hauss G, Bretthauer M, Sauar J, et al. Polyethylene glycol versus sodium phosphate in bowel cleansing for colonoscopy: a randomized trial. Endoscopy 2005;37:537-541.

16. DiPalma JA, Wolff BG, Meagher A, Cleveland M. Comparison of reduced volume versus four liters sulfate-free electrolyte lavage solutions for colonoscopy colon cleansing. Am J Gastroenterol 2003;98:2187-2191.

17. Ker TS. Comparison of reduced volume versus four-liter electrolyte lavage solutions for colon cleansing. Am Surg 2006;72:909-911.

18. Kao D, Lalor E, Sandha G, et al. A randomized controlled trial of four precolonoscopy bowel cleansing regimens. Can J Gastroenterol 2011;25:657-662.

19. Hookey LC, Depew WT, Vanner SJ. Combined low volume polyethylene glycol solution plus stimulant laxatives versus standard volume polyethylene glycol solution: a prospective, randomized study of colon cleansing before colonoscopy. Can J Gastroenterol 2006;20:101-105.

20. Golub RW, Kerner BA, Wise WE Jr, et al. Colonoscopic bowel preparations--which one? A blinded, prospective, randomized trial. Dis Colon Rectum 1995;38:594-599. 